\title{
3D Face Matching Using the Surface Interpenetration Measure
}

\author{
Olga R.P. Bellon, Luciano Silva, and Chauã C. Queirolo* \\ Universidade Federal do Paraná, Departamento de Informática, \\ IMAGO Research Group, CP 19081, 81531-980, Curitiba - PR, Brasil \\ \{olga, luciano, ccqs03\}@inf.ufpr.br \\ http://www.inf.ufpr.br/imago
}

\begin{abstract}
D face recognition has gained growing attention in the last years, mainly because both the limitations of $2 \mathrm{D}$ images and the advances in 3D imaging sensors. This paper proposes a novel approach to perform 3D face matching by using a new metric, called the Surface Interpenetration Measure (SIM). The experimental results include a comparison with a state-of-art work presented in the literature and show that the SIM is very discriminatory as confronted with other metrics. The experiments were performed using two different databases and the obtained results were quite similar, showing the robustness of our approach.
\end{abstract}

\section{Introduction}

Nowadays, the researches in 2D face recognition have reached a significant degree of success but it is well known that some drawbacks still remain [1]. A number of computer vision experts believe that these limitations could be solved by using 3D information. Therefore, 3D face recognition has gained growing attention in the last years. The advances in 3D imaging technology (e.g. Intl. Conf. on 3-D Digital Imaging and Modeling) also have played an important role in this scenario.

Some important but few, disperse contributions regarding 3D face processing were made in early 90's 2345]. In the last years, the contributions related to this subject have increased in number and more consistent experimental results have been presented, mostly based on private databases. For a survey of works presented in the literature about $3 \mathrm{D}$ face processing the reader should refers to $[$.

Basically, the main approaches for $3 \mathrm{D}$ face recognition are based in one or combinations of different techniques, including: image registration by the Iterative Closest Point (ICP) 7] algorithm; Extended Gaussian Image (EGI) 8]; and Principal Component Analysis (PCA) 9. Recently, ICP-based techniques have been developed [1011] to assess matching quality based on Root Mean Square Error (RMSE). Because the RMSE alone is not discriminatory enough [10] for the purpose, these works combine other information to achieve a final decision

\footnotetext{
* The authors would like to thank CNPq for financial support.
} 
about the recognition [10]. EGI-based techniques were more applied in the early 90's and present severe limitations regarding scale 312. Finally, the PCA family techniques have been extensively used for $2 \mathrm{D}$ face recognition but even by adding 3D information they still suffer from facial expression limitations and also there is the "curse of dimensionality". Multi-modal approaches have also applied PCA techniques [1314].

In this work we propose a new metric for the 3D face matching problem, based on the Surface Interpenetration Measure (SIM) [15,161718. The SIM has proved to be a robust measure for $3 \mathrm{D}$ image registration and the experiments presented in this paper shows that it is also suitable for $3 \mathrm{D}$ face matching. Even more, the SIM produces a better range for discrimination between faces as compared with other metrics. In order to prove that, we reproduced the metrics presented in [10] and included comparisons against the SIM. Our experiments were performed using two different databases and the obtained results validate the robustness of our approach, which may contribute substantially to this field.

This paper is organized as follows. First, we introduce our developed approach by using the Surface Interpenetration Measure (SIM) in section 2, The experimental results by comparing different measures for $3 \mathrm{D}$ face matching are presented in section 3 , followed by a conclusion in section 4 .

\section{Developed Approach Using the SIM}

We propose to perform 3D face matching based on our robust range image registration method which combines Genetic Algorithms (GA) and the Surface Interpenetration Measure (SIM) 15 16 17 18.

\subsection{The Surface Interpenetration Measure}

The SIM was developed by analyzing visual results of two aligned surfaces, each rendered in a different color, crossing over each other repeatedly in the overlapping area. The interpenetration effect results from the nature of real range data, which presents slightly rough surfaces with small local distortions caused by limitations of the acquiring system [15. Because of this, even flat surfaces present a "roughness" in range images. With this, we can assume that independently of the surfaces' shapes the interpenetration will always occur. We also observed that two views acquired from the same object surface with the same scanner position and parameters provide two different range images.

By quantifying interpenetration, one can more precisely evaluate registration results and provide a highly robust control. To do this we developed the following measure based on the surface normal vector, computed by a local least squares planar fit, at each point. After the alignment of two images, $A$ and $B$, we identify the set of interpenetrating points in $A$ with respect to $B$. For each point $p \in A$ we define a neighborhood $N_{p}$ to be a small $n \times n$ window centered on $p$. With $q$ denoting a point in the neighborhood $N_{p}, c$ the corresponding point of $p$ in image $B$ and $\boldsymbol{n}_{c}$ the local surface normal at $c$, we define the set of interpenetrating 
points as: $C_{(A, B)}=\left\{p \in A \mid\left[\left(\overrightarrow{q_{i}-c}\right) \cdot \boldsymbol{n}_{c}\right]\left[\left(\overrightarrow{q_{j}-c}\right) \cdot \boldsymbol{n}_{c}\right]<0\right\} ;$ where $q_{i}, q_{j} \in N_{p}$ and $i \neq j$. This set comprises those points in $A$ whose neighborhoods include at least one pair of points separated by the local tangent plane, computed at their correspondents in $B$. With this, we then define the SIM as the fraction of interpenetrating points in $A: \operatorname{SIM}_{(A, B)}=\frac{\left|C_{(A, B)}\right|}{|A|}$.

Registrations of two views presenting good interpenetration have high SIM's values. Our experimental results show that erroneous alignments produce low SIM's values and that small differences in MSE can yield significant differences in SIM. Furthermore, alignments with high SIM present a very low interpoint distance between the two surfaces. That is, the SIM is a far more sensitive indicator of alignment quality when comparing "reasonable" alignments [16 18].

\subsection{Robust GA-Based Approach for Range Image Registration}

The general principle underlying GA is to maintain a population of possible solutions (individuals) encoded in the form of a chromosome (a string of genes) and to submit the population to an evolutionary procedure, until some criteria can be satisfied [19]. In this process GA operators (e.g. crossover, mutation and selection) and a fitness function are used to define the best individuals for each generation. At the end of the evolutionary procedure we have the best individual.

Here the goal of the registration problem is to find through a pose-space search, rather than correspondence-base search of ICP, geometric transformations that can be used to align two views precisely. To perform range image registration using GA it is necessary to define a chromosome encoded as: 6 genes, 3 parameters each of rotation and translation [17. With this robust GA-based approach driven by the SIM we obtained precise alignments [15[16 18].

In this work we adopted the same strategy as in [16 18 to align views to allow a precise face matching. However, as shown in section 3, the SIM did not necessarily need our robust GA-based registrations to achieve discriminatory matching results, which can also be obtained from ICP-based registrations.

Fig. 11 shows examples of using face views from two different databases: Notre Dame 3D Face Database (NDU) 1 and OSU SAMPL Database (OSUD) 2 . The alignments shown in Figs. 1(c) and 1(i) were obtained using an ICP-based approach (see section 3 for details).

\section{Experimental Results for 3D Face Matching}

The main goal of our experiments was to validate the SIM as a novel measure for $3 \mathrm{D}$ face matching. For this, we use a number of different faces from two different range image databases. Each pair of views were aligned with with two different approaches (GA and ICP) to show the SIM's improvements and robustness. Also, we compare our measure against the ones proposed in [10].

\footnotetext{
${ }^{1}$ www.und.edu/ ${ }^{c v r l}$

2 sampl.eng.ohio-state.edu
} 


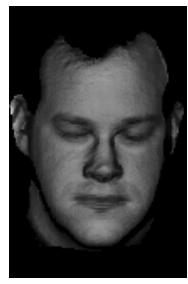

(a)

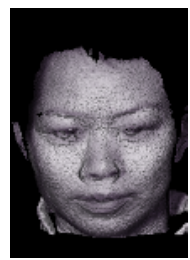

(g)

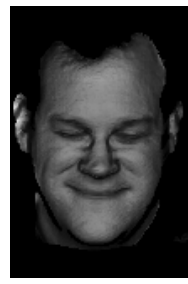

(b)

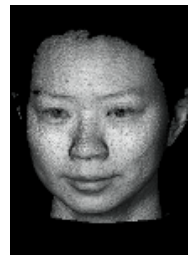

(h)

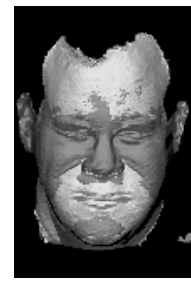

(c) 1.81

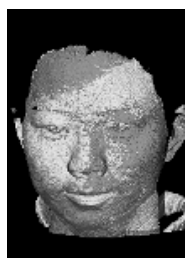

(i) 0.81

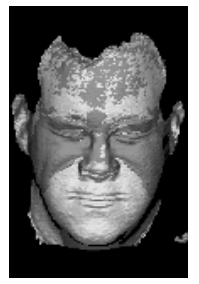

(d) 2.06

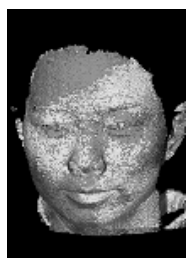

(j) 1.04

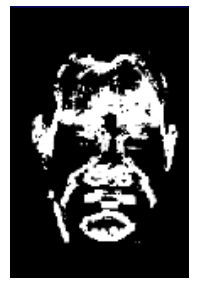

(e) $24 \%$

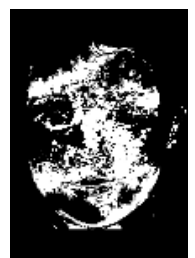

(k) $25 \%$

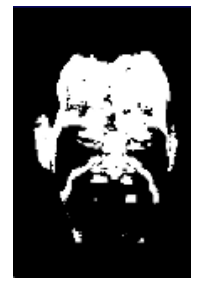

(f) $38 \%$

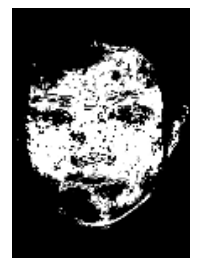

(1) $37 \%$

Fig. 1. Examples of SIM resuls: (a) and (b) views from OSUD; (g) and (h) views from $\mathrm{NDU}$; (c and i) and ( $\mathrm{d}$ and $\mathrm{j}$ ) are registration results with their RMSE values using ICP-based (c and i) and GA-based approaches ( $d$ and j), respectively; (e and k) and (f and 1 ) are the SIM values and the binary images of the interpenetrating points obtained from the alignments as show in (c and i) and ( $d$ and $j)$, respectively

Figs. 1(d) and 1(j) were obtained using our robust GA-based approach. As can be seen in Figs. 1(c) 1(d) and Figs. 1(i) 1(j) both aligned views show correct registrations having similar RMSE values. However, by analyzing the SIM values and the respective binary images obtained by our approach we obtained more precise alignments to improve the face matching problem.

From our best knowledge, there is no large public database of 3D faces. In our experiments, we used two databases: the Notre Dame 3D Face Database (NDU) and the OSU SAMPL Database (OSU). From the NDU database, we used only the portion of the image that represents the face to obtain precise measures during our experiments. This database includes 200 subjects with different views and all of them were acquired in frontal view and with neutral expression. In our experiments, we selected a set of 2 views from each one of the 5 subjects (A-E), as shown in Fig. 2, The image dimensions are 640x480, obtained by a Minolta Vivid 900 range scanner (wWw.minoltausa.com/vivid).

From the OSU database, we selected 6 subjects (A-F) as presented in Fig. 3 . two of them (A and B) have 3 images with neutral facial expression acquired at 36 degree spacing as shown in Figs. 3(a) 3(c) and Figs. 3(h) $3(\mathrm{j})$ respectively; and 2 images with smiling facial expression (Figs. $3(\mathrm{~d}), 3(\mathrm{e}) \mid$ and Figs. $3(\mathrm{k}) \mid 3(\mathrm{l})]$; and 2 images with sad facial expression (Figs. $3(\mathrm{f})+3(\mathrm{~g})$ and Figs. $3(\mathrm{~m}) \cdot 3(\mathrm{n})$ ) . All the remaining have 1 frontal image with neutral expression, as in Figs. $3(\mathrm{o}) \mid 3(\mathrm{r})$ The image dimensions are 200x200 obtained by a Minolta Vivid 700.

\footnotetext{
${ }^{3}$ The authors would like to thank the databases owners for allowing us to use them.
} 


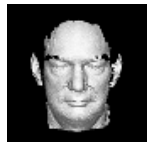

(a) $\mathrm{A0}$

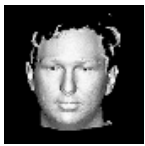

(f) $\mathrm{C} 1$

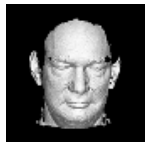

(b) A1

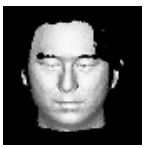

(g) D0

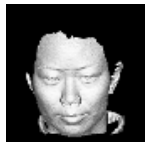

(c) B0

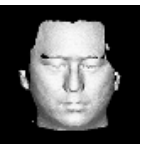

(h) D1

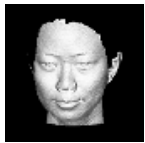

(d) B1

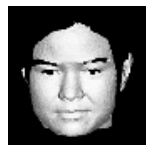

(i) E0

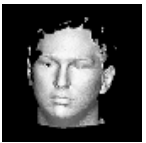

(e) $\mathrm{CO}$

Fig. 2. Faces from the Notre Dame 3D Face Database

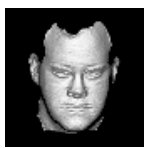

(a) $\mathrm{A} 0$

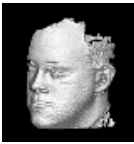

(g) $\mathrm{A} 6$

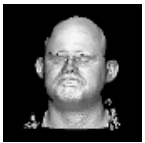

(m) B5

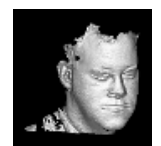

(b) A1

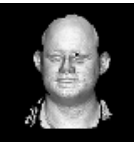

(h) B0

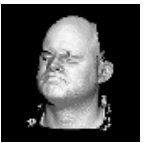

(n) B6

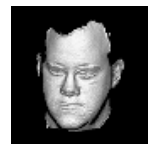

(c) A2

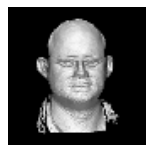

(i) $\mathrm{B} 1$

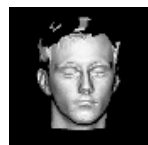

(o) $\mathrm{C}$

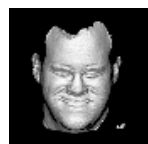

(d) A3

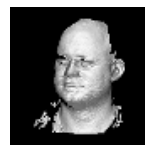

(j) B2

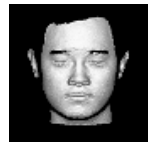

(p) D

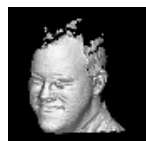

(e) A4

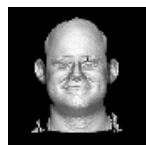

(k) B3

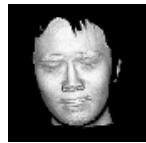

(q) $\mathrm{E}$

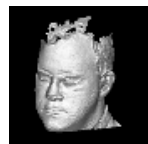

(f) $\mathrm{A} 5$

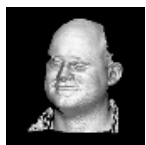

(l) B4

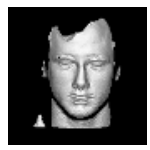

(r) $\mathrm{F}$

Fig. 3. Faces from the OSU SAMPL Database

Because the range views from both databases were not registered we had to align each pair of views used in our experiments by using two different algorithms: 1) ICP-based approach driven by the RMSE measure used in the Scanalyze Software (graphics.stanford.edu/software); and 2) our robust GAbased approach driven by the SIM as presented in [16 18,15].

With the obtained registrations we reproduced the metrics presented in [10], which use a combination of RMSE and cross correlation of the shape index vectors [20] as a face matching measure. Also, the registrations were used to compare the SIM against different metrics as presented bellow.

First, from each aligned view pair the region $R$ around eyes and nose (see Fig. 4(a) was manually extracted because it is considered rigid, suffering less influence from facial expressions 21. Then, from each region we selected a set $S_{p t s}$ of control points (around 100) equally spaced, as shown in Fig.4(b). For both $R$ and Spts we considered only valid corresponding points within the overlap area to compute the metrics as in [10]. 


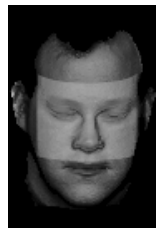

(a)

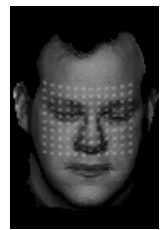

(b)

Fig. 4. Regions of interest used to compute the matching measures as in [10]: (a) region $R$ around eyes and nose; (b) set $S_{p t s}$ of control points

The results in applying the approach proposed in [10 on views from both databases are presented in Tables 1 and 2, respectively. The first two columns of the tables (V1 and V2) represent each aligned pair (e.g. view A0 aligned to A1). M1 represents the global RMSE of the alignment; M2 is cross correlation of the shape index vectors and M3 the face matching measure as proposed by [10], which is the combination of M2 and the RMSE in region $R$. The RMSE in the region $R$ can be known by: $M_{R}=M 3-M 2$.

As suggested in [22], a pre-defined threshold $\delta=1$ should be used to classify correct and incorrect matchings. If $M 3<\delta$ than we have a correct matching, i.e. alignment A0-A1 from Table 1; otherwise the aligment represents an incorrect matching, i.e. aligment B0-E1 from Table 1. However, it is well known that threshold definition is not a trivial process. We observed that in some cases wrong matching occurs (e.g. alignments B0-B1 and B0-B2 from Table 2), which are faces from the same subject with neutral expression. Also, because the range between correct and incorrect matchings tends to be lower it is hard to define a good discriminant threshold.

By analysing M3 values we observed that the distance metrics used in this approach fails in detecting correct matchings for faces of a same subject but with different facial expression. For instance in the Table 2, the M3=1.58 of alignment B0-A1 (distinct subjects) should be higher than the results for the alignment A0-A3 (same subject), which have M3=1.85. In contrast, for the SIM, the difference between correct and incorrect matching values are much higher (for instance alignments $\mathrm{C} 0-\mathrm{C} 1$ and B0-E1 from Table 1). Also, the correct matches for two images of the same subject but with different facial expressions have an intermediate value between the correct and incorrect matches, suggesting that our matching measure can deal with changes in facial expressions.

We also performed a number of experiments to evaluate the SIM's results in terms of ICP-based and GA-based alignments. Tables 1 and 2 show the results of the SIM for both approaches: columns S1 and S1g presents the SIM's results in the region $R$ for the ICP-based and GA-based alignments, respectively. From the SIM's equation A and B represent the views V1 and V2, respectively. The columns S2 and S2g present the global SIM values of the alignments. As can be seen, the results generated by the ICP-based approach are quite similar from those obtained by applying our GA-based approach, proving that the applied metric (S1 or M3) is more relevant than the registration technique. 
Table 1. Experimental results from the Notre Dame 3D Database

\begin{tabular}{|c|c||c|c|c|c|c|c|c|}
\hline V1 & V2 & M1 & M2 & M3 & S1 & S1g & S2 & S2g \\
\hline \hline A0 & A1 & 0.85 & 0.04 & 0.72 & 27.4 & 29.0 & 24.7 & 29.8 \\
\hline B0 & B1 & 0.81 & 0.04 & 0.67 & 30.0 & 58.7 & 24.9 & 36.7 \\
\hline C0 & C1 & 0.98 & 0.02 & 0.84 & 26.3 & 38.4 & 22.6 & 30.8 \\
\hline D0 & D1 & 0.65 & 0.04 & 0.64 & 28.5 & 31.6 & 30.5 & 36.3 \\
\hline E0 & E1 & 0.68 & 0.04 & 0.57 & 42.1 & 42.4 & 47.3 & 49.1 \\
\hline A0 & B1 & 2.18 & 0.08 & 2.25 & 0.62 & 0.89 & 2.19 & 3.67 \\
\hline A0 & C1 & 2.24 & 0.07 & 2.08 & 4.50 & 6.82 & 3.64 & 8.58 \\
\hline A0 & D1 & 2.28 & 0.06 & 2.25 & 1.82 & 6.39 & 3.63 & 8.26 \\
\hline A0 & E1 & 2.43 & 0.08 & 2.42 & 1.85 & 1.64 & 1.47 & 2.22 \\
\hline B0 & A1 & 2.23 & 0.09 & 2.60 & 1.38 & 2.83 & 3.19 & 5.52 \\
\hline B0 & C1 & 2.37 & 0.10 & 2.34 & 1.35 & 3.64 & 3.24 & 6.51 \\
\hline B0 & D1 & 2.34 & 0.05 & 3.20 & 1.14 & 7.71 & 2.83 & 6.63 \\
\hline B0 & E1 & 2.02 & 0.06 & 1.24 & 7.87 & 10.1 & 6.51 & 10.2 \\
\hline C0 & A1 & 2.16 & 0.08 & 1.94 & 5.92 & 3.52 & 4.95 & 7.71 \\
\hline C0 & B1 & 2.65 & 0.11 & 2.75 & 0.10 & 0.54 & 1.40 & 2.78 \\
\hline C0 & D1 & 1.74 & 0.05 & 1.97 & 6.47 & 6.02 & 8.31 & 9.19 \\
\hline C0 & E1 & 2.35 & 0.06 & 1.42 & 1.02 & 7.14 & 1.07 & 3.88 \\
\hline D0 & A1 & 2.17 & 0.07 & 1.67 & 1.13 & 3.78 & 3.30 & 7.88 \\
\hline D0 & B1 & 2.42 & 0.05 & 2.04 & 2.76 & 1.50 & 1.89 & 4.37 \\
\hline D0 & C1 & 1.78 & 0.08 & 1.91 & 4.91 & 10.7 & 7.18 & 10.3 \\
\hline D0 & E1 & 2.12 & 0.13 & 2.51 & 2.69 & 6.04 & 2.42 & 4.73 \\
\hline E0 & A1 & 2.37 & 0.13 & 2.09 & 1.70 & 5.25 & 1.96 & 3.49 \\
\hline E0 & B1 & 1.96 & 0.11 & 2.28 & 6.54 & 7.66 & 5.13 & 12.9 \\
\hline E0 & C1 & 2.15 & 0.06 & 2.40 & 4.74 & 7.73 & 4.66 & 7.25 \\
\hline E0 & D1 & 2.15 & 0.04 & 1.82 & 3.98 & 2.66 & 4.60 & 6.43 \\
\hline
\end{tabular}

Table 2. Experimental results from the OSU SAMPL Database

\begin{tabular}{|l|l||l|l|l|l|l|l|l|}
\hline V1 & V2 & M1 & M2 & M3 & S1 & S1g & S2 & S2g \\
\hline \hline A0 & A1 & 0.99 & 0.01 & 0.93 & 59.5 & 62.2 & 57.6 & 60.8 \\
\hline A0 & A2 & 0.85 & 0.03 & 0.93 & 71.7 & 72.0 & 62.8 & 65.5 \\
\hline B0 & B1 & 1.04 & 0.01 & 1.14 & 62.2 & 63.8 & 67.8 & 68.4 \\
\hline B0 & B2 & 1.05 & 0.01 & 1.04 & 53.6 & 51.5 & 55.4 & 57.8 \\
\hline A0 & A3 & 1.81 & 0.06 & 1.85 & 27.3 & 35.4 & 23.7 & 37.7 \\
\hline A0 & A4 & 1.85 & 0.06 & 2.08 & 18.5 & 21.0 & 17.4 & 21.6 \\
\hline A0 & A5 & 1.27 & 0.04 & 1.45 & 31.0 & 36.9 & 32.5 & 35.8 \\
\hline A0 & A6 & 1.20 & 0.02 & 1.68 & 28.0 & 32.4 & 27.4 & 32.8 \\
\hline B0 & B3 & 1.70 & 0.02 & 1.78 & 34.3 & 32.5 & 30.0 & 33.5 \\
\hline B0 & B4 & 1.69 & 0.03 & 1.85 & 25.4 & 32.3 & 20.5 & 28.5 \\
\hline B0 & B5 & 1.91 & 0.04 & 1.87 & 35.0 & 51.2 & 24.7 & 34.3 \\
\hline B0 & B6 & 1.93 & 0.02 & 1.77 & 25.4 & 36.5 & 18.9 & 26.3 \\
\hline A0 & B0 & 2.16 & 0.11 & 2.70 & 9.07 & 10.6 & 12.2 & 12.5 \\
\hline A0 & B1 & 2.08 & 0.13 & 2.61 & 9.97 & 8.57 & 11.7 & 13.6 \\
\hline A0 & B2 & 2.12 & 0.08 & 2.77 & 7.02 & 9.69 & 9.81 & 11.1 \\
\hline A0 & C & 2.21 & 0.10 & 2.31 & 6.33 & 11.0 & 5.53 & 7.59 \\
\hline A0 & D & 2.25 & 0.10 & 2.43 & 8.23 & 5.98 & 5.47 & 8.09 \\
\hline A0 & E & 2.18 & 0.08 & 2.27 & 4.19 & 7.34 & 4.33 & 6.56 \\
\hline A0 & F & 2.21 & 0.12 & 2.69 & 10.7 & 8.95 & 8.75 & 13.5 \\
\hline B0 & A1 & 2.39 & 0.06 & 1.58 & 4.32 & 6.78 & 7.11 & 8.09 \\
\hline B0 & A2 & 2.17 & 0.05 & 1.61 & 9.57 & 9.41 & 10.5 & 11.4 \\
\hline B0 & C & 2.65 & 0.11 & 2.88 & 1.69 & 1.71 & 3.32 & 4.61 \\
\hline B0 & D & 2.44 & 0.07 & 2.16 & 3.10 & 9.32 & 3.46 & 6.64 \\
\hline B0 & E & 2.66 & 0.11 & 2.98 & 1.90 & 2.01 & 4.64 & 5.06 \\
\hline B0 & F & 2.19 & 0.05 & 2.07 & 9.16 & 10.2 & 10.7 & 12.9 \\
\hline
\end{tabular}

From the tables, one can see that for the RMSE values the range between a correct matching (views form the same subject) and an incorrect one (views from different subjects) is very small, although they fit the values specified in 10.

\section{Conclusion}

In this paper we introduce a novel and robust approach for 3D face matching by using the Surface Interpenetration Measure. We observed that the SIM is a reliable, discriminant measure that can deal better with changes in facial expressions as compared with other measures (i.e. M3). Although the SIM's results from our GA-based approach have presented better results, we proved that the SIM, when used as a face matching measure, works even with ICP-based alignments. As a future work we plan to use the SIM to identify face expressions and to develop a robust approach for 3D face identification problem using a larger database.

\section{References}

1. Zhao, W., Chellappa, R., Phillips, P.J., Rosenfeld, A.: Face recognition: A literature survey. ACM Computing Surveys 35 (2003) 399-458

2. Achermann, B., Jiang, X., Bunke, H.: Face recognition using range images. In: Intl. Conf. on Virtual Systems and Multimedia. (1997) 129-136

3. Lee, J.C., Milios, E.: Matching range images of human faces. In: Intl. Conf. on Computer Vision. (1990) 722-726 
4. Negamine, T., Uemura, T., Masuda, I.: 3d facial image analysis for human identification. In: Prof. of ICPR. (1992) 324-327

5. Brunelli, R., Falavigna, D.: Person identification using multiple cues. IEEE PAMI 17 (1995) 955-966

6. Bowyer, K.W., Chang, K.I., Flynn, P.J.: A survey of approaches to threedimensional face recognition. In: Proc. of ICPR. (2004) 358-361

7. Besl, P.J., McKay, N.D.: A method for registration of 3-D shapes. IEEE PAMI 14 (1992) 239-256

8. Horn, B.K.P.: Extended gaussian images. In: DARPA84. (1984) 72-89

9. Martinez, A.M., Kak, A.C.: Pca versus lda. IEEE PAMI 23 (2001) 228-223

10. Lu, X., Colbry, D., Jain, A.K.: Three-dimensional model based face recognition. In: Proc. of ICPR. (2004) 362-366

11. Cook, J., Chandran, V., Sridharan, S., Fookes, C.: Face recognition from 3d data using iterative closest point algorithm and gaussian mixture models. In: 3D Data Processing, Visualization, and Transmission. (2004) 502-509

12. Tanaka, H.T., Ikeda, M.: Curvature-based face surface recognition using spherical correlation - principal directions for curved object recognition. In: ICPR. Volume 3. (1996) 638-642

13. Chang, K.I., Bowyer, K.W., Flynn, P.J.: An evaluation of multimodal $2 \mathrm{~d}+3 \mathrm{~d}$ face biometrics. IEEE PAMI 27 (to appear in 2005) 619-624

14. Chang, K.I., Bowyer, K.W., Flynn, P.J.: Multi-modal 2d and 3d biometrics for face recognition. In: Intl. Workshop on Analysis and Modeling of Faces and Gestures. (2003) 187-194

15. Silva, L., Bellon, O.R.P., Boyer, K.L.: Robust Range Image Registration Using Genetic Algorithms and the Surface Interpenetration Measure. Volume 60 of Machine Perception and Artificial Intelligence. World Scientific Publishing (2005)

16. Silva, L., Bellon, O.R.P., Boyer, K.L.: Robust range image registration using the surface interpenetration measure and enhanced genetic algorithms. IEEE PAMI 27 (2005) 762-776

17. Silva, L., Bellon, O.R.P., Gotardo, P.F.U., Boyer, K.L.: Range image registration using enhanced genetic algorithms. In: IEEE International Conference on Image Processing. Volume 2. (2003) 711-714

18. Silva, L., Bellon, O.R.P., Boyer, K.L.: Robust multiview range image registration. In: Proc. of 17th Brazilian Symposium on Computer Graphics and Image Processing. (2003) 80-88

19. Man, K.F., Tang, K.S., Kwong, S.: Genetic algorithms: concepts and applications. IEEE Trans. on Industrial Eletronics 43 (1996) 519-534

20. Dorai, C., Jain, A.K.: COSMOS - a representation scheme for $3 \mathrm{~d}$ free-form objects. IEEE PAMI 19 (1997) 1115-1130

21. Colbry, D., Lu, X., Jain, A.K., Stockman, G.: 3d face feature extraction for recognition. Technical Report 4-39, MSU-CSE (2004)

22. Lu, X., Jain, A.K.: Integrating range and texture information for $3 \mathrm{~d}$ face recognition. In: IEEE Workshop on Applications of Computer Vision. (2005) 156-163 